\title{
Minimally invasive transforaminal lumbar interbody fusion with reduction of spondylolisthesis: technique and outcomes after a minimum of 2 years' follow-up
}

\author{
Paul Park, M.D., ${ }^{1}$ ANd Kevin T. Foley, M.D. ${ }^{2}$ \\ ${ }^{1}$ Department of Neurosurgery, University of Michigan Health System, Ann Arbor, Michigan; and \\ ${ }^{2}$ Semmes-Murphey Clinic and Department of Neurosurgery, University of Tennessee, Memphis, \\ Tennessee
}

\begin{abstract}
Minimally invasive transforaminal lumbar interbody fusion (MI-TLIF) is a relatively new surgical procedure that appears to minimize iatrogenic soft tissue and muscle injury. The authors describe a technique for MI-TLIF that permits the surgeon to reduce spondylolisthesis percutaneously. The results in 40 consecutive patients who underwent MITLIF for symptomatic spondylolisthesis utilizing this approach are reviewed. Thirty cases involved a degenerative spondylolisthesis while the remaining 10 were isthmic. The minimum follow-up was 24 months with a mean of 35 months. The mean preoperative Oswestry Disability Index score was 55, decreasing to a mean of 16 postoperatively. The mean leg and back pain visual analog scale scores were 65 and 52, respectively, improving to means of 8 and 15 . Reduction of the spondylolisthesis was achieved in all cases, with a mean decrease in forward translation of $76 \%$. The authors conclude that MI-TLIF for symptomatic spondylolisthesis appears to be an effective surgical option with results that compare favorably to open procedures. (DOI: $10.3171 / \mathrm{FOC} / 2008 / 25 / 8 / \mathrm{E} 16$ )
\end{abstract}

\section{KEY WORDS • minimally invasive surgery • reduction • spondylolisthesis • transforaminal lumbar interbody fusion}

$\mathrm{T}$ RANSFORAMINAL lumbar interbody fusion has become increasingly popular when arthrodesis is chosen to treat mechanical back pain caused by degenerative disc disease and/or spondylolisthesis. It offers the advantage of obtaining a circumferential arthrodesis via a unilateral approach with minimal retraction of neural elements. ${ }^{8,12}$ When compared to the open procedure, MI-TLIF appears to achieve similar rates of arthrodesis while minimizing iatrogenic soft tissue/muscle injury and blood loss. ${ }^{3,14}$ Shorter hospital stays and decreased narcotic usage also appear to be advantages of MI-TLIF. ${ }^{6}$ While a variety of techniques for open spondylolisthesis reduction have been described, ${ }^{1,5,11}$ the technical aspects involved in minimally invasive spondylolisthesis reduction have not been well described. We present a technique for MI-TLIF that permits the surgeon to reduce spondylolisthesis percutaneously, utilizing translational screw extenders. In addition, the clinical outcomes of a consecutive series of patients treated for symptomatic spondylolisthesis in this manner are reported.

Abbreviations used in this paper: MI-TLIF = minimally invasive transforaminal lumbar interbody fusion; ODI = Oswestry Disability Index; rhBMP = recombinant human bone morphogenetic protein; VAS $=$ visual analog scale.

\section{Methods}

Study Design

The study was a retrospective chart review. The study population included all patients with symptomatic degenerative or isthmic spondylolisthesis who underwent MI-TLIF between January 2002 and April 2006. Forty consecutive patients were identified, and all were included in the study. Demographic characteristics, surgical data, radiographic studies, and functional outcome data were recorded.

\section{Outcome Assessment}

Pain and functional disability were quantitatively measured using a VAS and the ODI. The VAS scores were recorded on a $100-\mathrm{mm}$ horizontal line with 0 equal to "no pain," and 100 equal to "very severe pain." The ODI was scored on a 0-100 scale using the Oswestry Low Back Pain Disability Questionnaire. On this scale, 0-20 equates to minimal disability, 20-40 moderate disability, 40-60 severe disability, 60-80 crippled, and 80-100 bed-bound or exaggerating. ${ }^{2}$ Both the VAS and ODI were prospectively acquired.

\section{Surgical Technique}

Surgical access for interbody fusion was obtained using 


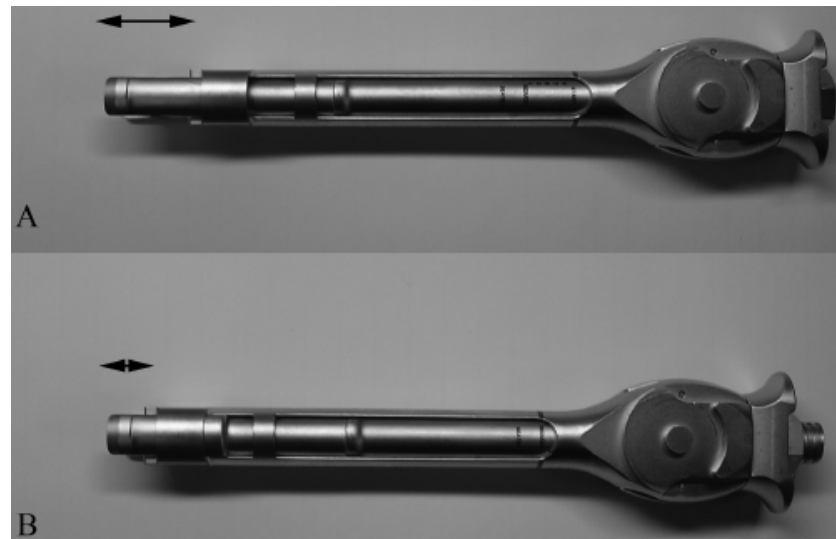

FIG. 1. Photographs of the reduction screw extender with the inner adjustable sleeve. Movement of the inner sleeve to the "reduced" position (B) pulls the screw head to the rod. (See also Fig. 3.)

a tubular retraction system (METRx, Medtronic Sofamor Danek). Pedicle screws and rods were placed percutaneously (CD Horizon Sextant, Medtronic Sofamor Danek). A newly designed reduction screw extender (Figs. 1 and 2) allowed for spondylolisthesis reduction by incorporating an adjustable inner sleeve. This sleeve, which attaches to the pedicle screw inserted into the listhesed vertebral body, can translate up to $2 \mathrm{~cm}$ relative to the extender body. After a screw has been inserted into the nondisplaced adjacent vertebral body and a percutaneously inserted rod has been locked to the nondisplaced screw, the driving mechanism on the reduction extender is slowly turned. This maneuver pulls the inner sleeve and the screw attached to the listhesed vertebral body back towards the rod, reducing the spondylolisthesis (Fig. 3).

In each case the patient was positioned prone on a Jackson spinal surgery table. In some cases, positioning alone resulted in some degree of postural reduction. A midline mark was drawn on the dorsal skin surface. Parallel lines were then drawn $4-4.5 \mathrm{~cm}$ from the midline mark on either side. The MI-TLIF approach was carried out from the side that was most symptomatic. Under fluoroscopic guidance, a 22-gauge spinal needle was used to determine the skin entry point and the trajectory to the appropriate interspace. Once an appropriate entry point and trajectory were determined, a 1-inch incision was centered on that point. A K-wire was then advanced until the fascia was pierced. Sequential dilation was performed and a 22-mmdiameter tubular retractor of appropriate length was placed (Fig. 4).

Under microscopic visualization, a high-speed drill was used to make a cut through the lamina, just medial to the facet joint. The cut was extended cranially to the level of the pars, at which point a transverse cut was made through the pars. Once these cuts were made, the inferior articular process was "free floating," and it was then removed and saved as autograft. The remaining superior articular process was also resected, as was the underlying ligamentum flavum, exposing the dural sac and the lateral edge of the traversing nerve root. If a more central and/or contralateral decompression were desired (as for concomitant spinal stenosis), the tubular retractor was "wanded" medially. The

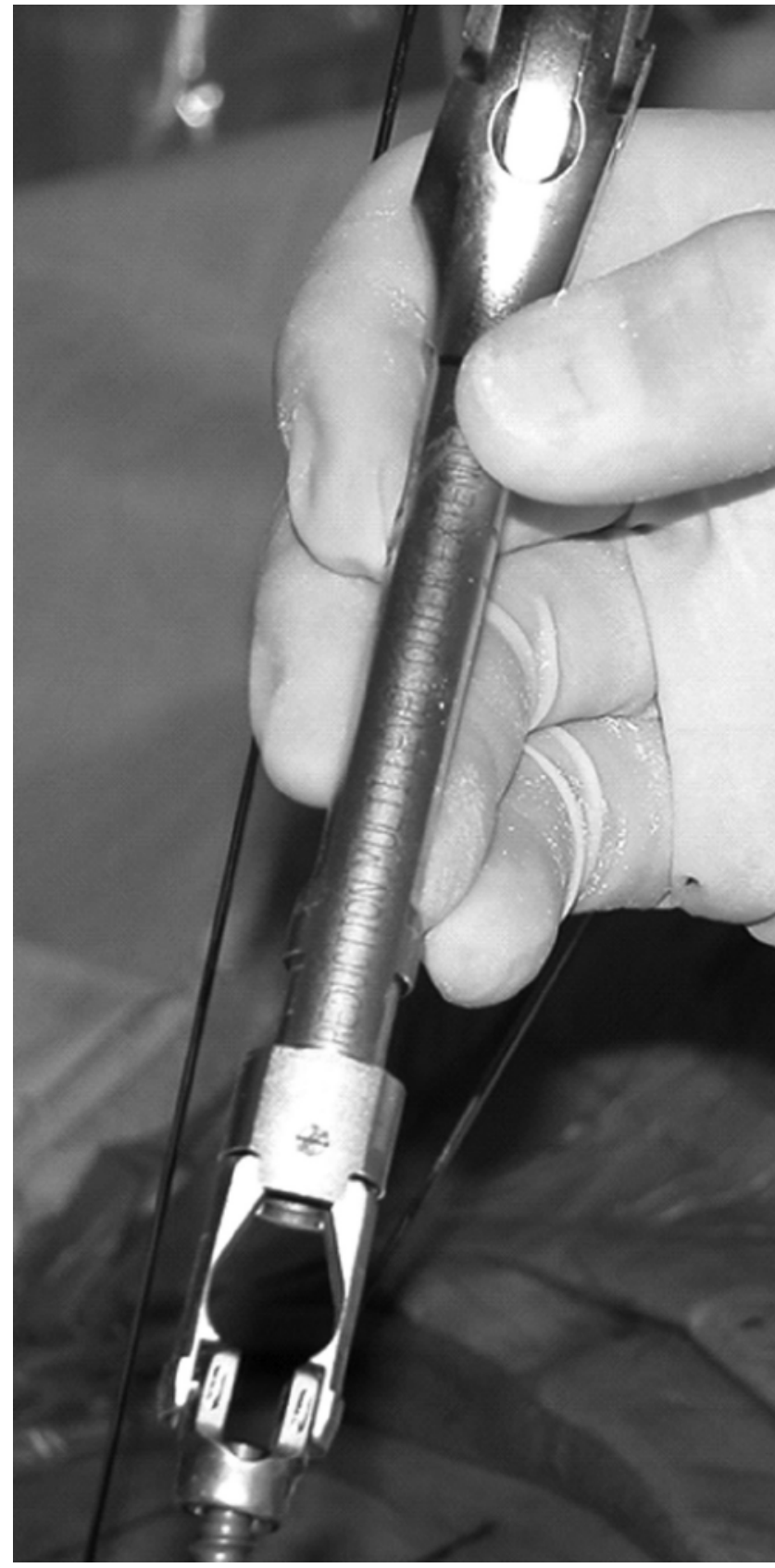

FIG. 2. Photograph of the reduction screw extender attached to a screw. The screw extender is in the unreduced position, as evidenced by the large offset of the apex of the screw extender aperture from the base of the screw head.

drill was then used to undercut the contralateral lamina and the underlying ligamentum flavum was removed, as necessary.

Once an adequate decompression was obtained, a complete discectomy was performed. If the disc space was collapsed, a small osteotome was used to initially open it. A distracting osteotome was then employed to further open the disc space so that sequential intervertebral distractors could be used to restore disc height (Fig. 5). Of note, distraction of the disc space often resulted in some measure of spondylolisthesis reduction. This technique for interbody 


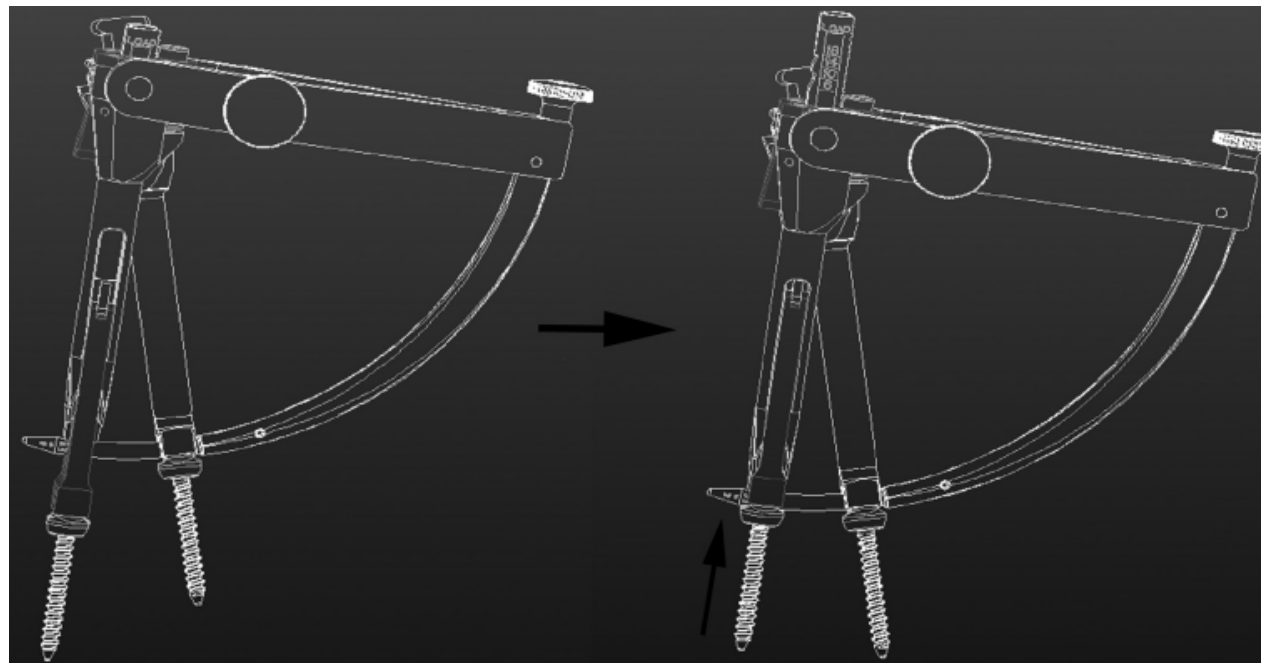

FIG. 3. Diagram showing how reduction is accomplished by shortening the length of the reduction extender. When the set screw of the caudal extender is provisionally tightened, this locks the angle between the rod and the caudal pedicle screw. Turning the drive mechanism of the reduction extender pulls the inner sleeve and the screw attached to the listhesed vertebral body back toward the rod, reducing the spondylolisthesis.

distraction and reduction utilizing sequentially larger dilators was previously described by Mummaneni and Rodts. ${ }^{10}$

After disc space distraction, attention was turned to the contralateral side for pedicle screw placement. Using the previously drawn line $4-4.5 \mathrm{~cm}$ from the midline, two $22-$ gauge spinal needles were used to determine entry points for placement of the pedicle screws. A 1-inch incision was centered between the entry points of the spinal needles. Under anteroposterior and lateral fluoroscopic guidance, each pedicle was traversed with a Jamshidi needle followed by placement of a K-wire and needle withdrawal. At this point, if adequate spondylolisthesis reduction had been accomplished with patient positioning and/or distraction of

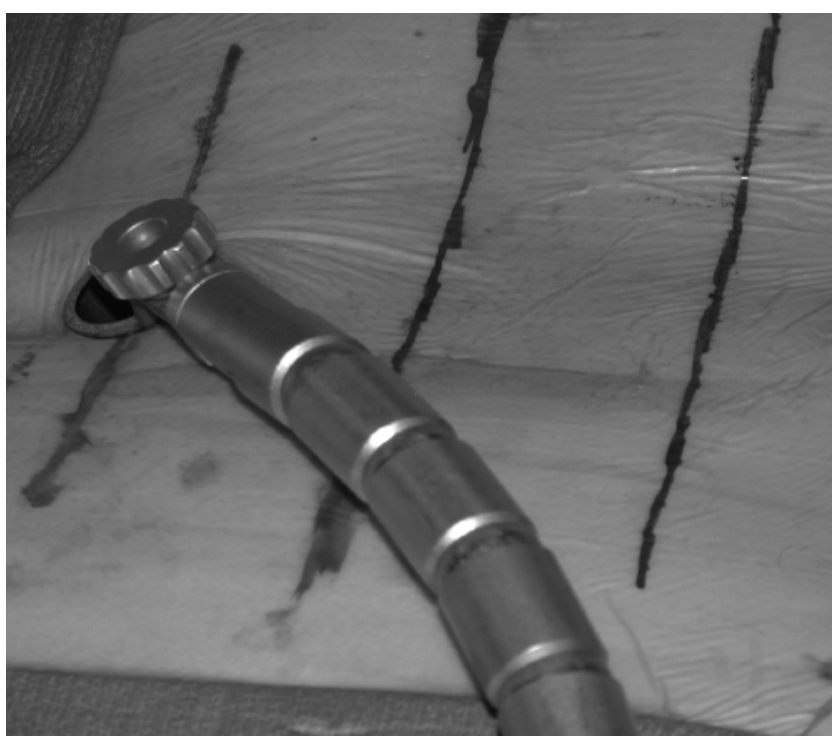

FIG. 4. Photograph showing proper placement of the tubular retractor centered over the disc space via a 1-inch incision. Note that a midline mark has been drawn with parallel lines demarcated $4.5 \mathrm{~cm}$ from the midline. the disc space, appropriately sized pedicle screws were inserted followed by the placement of a percutaneous rod. If, however, further spondylolisthesis reduction was desired, appropriately sized screws were loaded into the reduction screw extenders. Based on the amount of reduction desired, the inner sleeve of the cephalad screw extender was adjusted. Once the screws had been inserted into the pedicles, a rod of appropriate length was positioned via the percutaneous arc. The screw extenders were aligned in the sagittal plane so that the force applied by the reduction screw extender resulted in posterior translation without rotation of the listhesed vertebral body. The variable sleeve of the caudal screw extender was adjusted to the "reduced" position, and a set screw was placed into the caudal pedicle screw head, fixating the rod into a position offset from the cephalad screw head (Fig. 6). Shortening the sleeve of the cephalad screw extender pulled the screw head to the rod, thereby reducing the spondylolisthesis and allowing the placement of a locking set screw. Multiple fluoroscopic images were obtained during reduction. Of note, even in patients with Grade II spondylolisthesis, an attempt was made to fully reduce the slippage as long as adequate disc space distraction had been achieved.

Attention was then returned to the ipsilateral side, where the intervertebral distractor was removed. Distraction was maintained by the contralateral pedicle screw and rod construct (Fig. 6D). The empty disc space was filled initially with rhBMP-2 soaked in a collagen sponge carrier as well as local autograft, leaving a channel for an interbody implant. An appropriately sized polyetheretherketone (PEEK) interbody implant was selected and filled with rhBMP-2 and autograft. Because of the oblique trajectory afforded by starting $4-4.5 \mathrm{~cm}$ off the midline, the interbody implant could be placed so as to cross the midline of the disc space. Once the graft was placed, attention was returned to the contralateral side where the cephalad locking screw was loosened. The screw extenders were compressed to produce segmental lordosis, and final tightening of the locking screws was carried out. The tubular retractor was removed, 


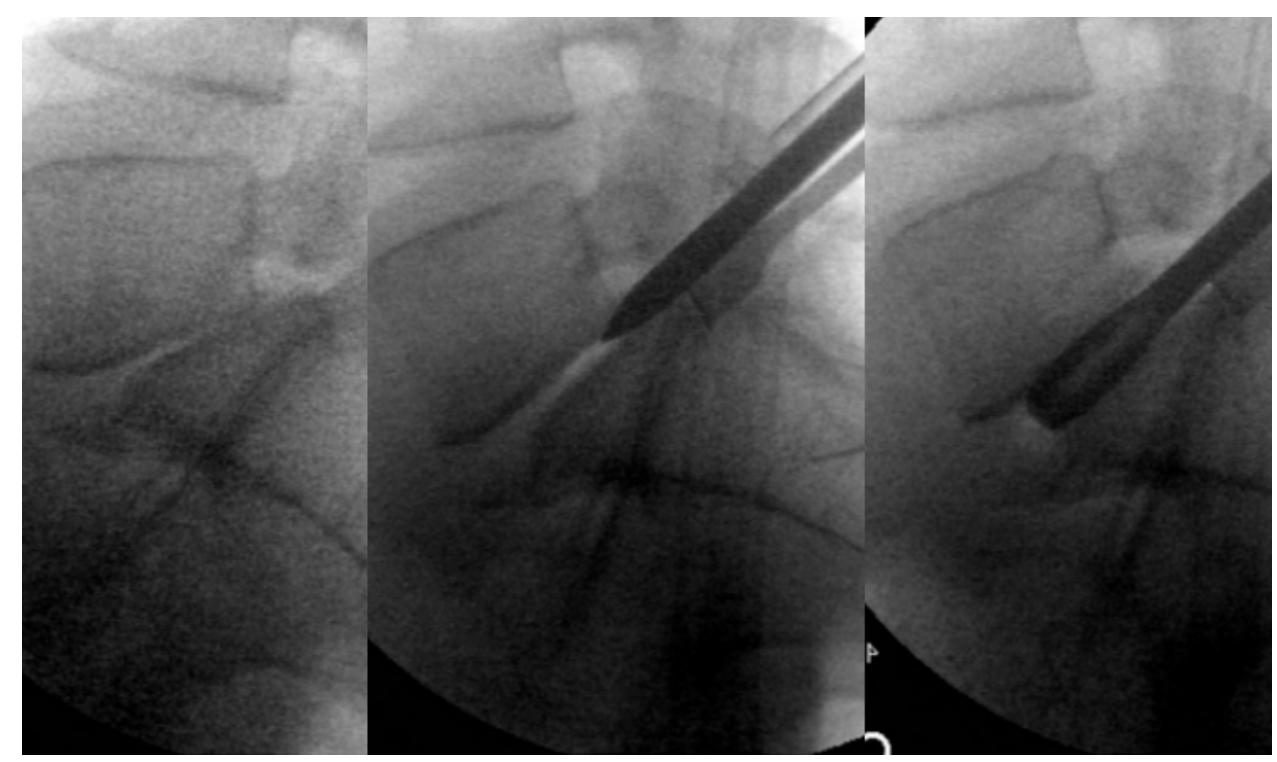

FIG. 5. Lateral fluoroscopic images showing restoration of disc height. A distracting osteotome is used to enter the disc space and obtain enough height to allow placement of the initial distractor, followed by sequentially larger interbody distractors. Note that in this case, there is minimal spondylolisthesis reduction with distraction.

and, using the same 1-inch incision, percutaneous pedicle screws were placed on the ipsilateral side. Although it is possible to place the ipsilateral screws under direct visualization, a larger retractor is typically required. To minimize incision size and consequent adjacent tissue injury, our preference was to remove the tubular retractor and place screws percutaneously. After placement of the screws, a percutaneous rod was positioned into place via the arc, the screw extenders were compressed, and final tightening of the last set of locking screws was performed.

\section{Results}

Of the 40 patients in the clinical series, $21(52.5 \%)$ were men; the mean age was 56 years (range $38-71$ years, Table 1). Thirty patients carried a diagnosis of degenerative spondylolisthesis and the remaining 10 had isthmic spondylolisthesis. Thirty-two patients had a Meyerding Grade I spondylolisthesis, whereas 8 had a Grade II slippage. The most common level treated was L4-5 (28 patients) followed by L5-S1 (8 patients) and then L3-4 (2 patients). Two patients underwent a 2-level fusion involving L4-S1. All patients underwent MI-TLIF with bilateral percutaneous fixation (Fig. 7). There were no conversions to an open procedure.

The mean duration of follow-up was 35 months (range 24-60 months). The mean leg pain VAS score for leg pain was 65 (range 9-99) preoperatively and decreased to 8 (range 0-22) at 2-year follow-up. The mean VAS score for back pain was 52 (range 0-97) preoperatively and decreased to 15 (range $0-36$ ) at 2 years postoperatively. The mean ODI score was 55 (range 44-80) preoperatively, whereas at 2-year follow-up it was 16 (range 2-38). A partial or complete reduction was accomplished in all cases. The mean translational reduction was $76 \%$ based on review of preoperative and postoperative radiographs. Postoperative CT was obtained at 2-year follow-up in all patients.
Fusion defined by bridging bone (Fig. 8) was demonstrated in all cases.

\section{Discussion}

A number of studies have evaluated the outcomes of posterolateral fusion for the treatment of spondylolisthesis. Ghogawala et al. ${ }^{4}$ reviewed 34 cases involving patients with degenerative Grade I spondylolisthesis, 14 of whom underwent laminectomy and posterolateral fusion and 20 of whom underwent laminectomy alone. In the fusion subgroup, the preoperative ODI and SF-36 physical component summary scores were 41.5 and 29.8 , respectively,

\section{TABLE 1}

Patient characteristics and outcomes of MI-TLIF with spondylolisthesis reduction

\begin{tabular}{ll}
\hline \hline \multicolumn{1}{c}{ Variable } & \multicolumn{1}{c}{ Value } \\
\hline no. of patients & 40 \\
mean age (range) & 56 yrs (38-71 yrs) \\
sex & $21(52.5)$ \\
$\mathrm{M}(\%)$ & $19(47.5)$ \\
$\mathrm{F}(\%)$ & $32(80)$ \\
Meyerding grade & $8(20)$ \\
I (\%) & 76 \\
II $(\%)$ & 35 mos $(24-60$ mos) \\
mean reduction (\%) & $65(9-99)$ \\
mean follow-up (range) & $8(0-22)$ \\
leg pain & \\
mean preop VAS score (range) & $52(0-97)$ \\
mean postop VAS score (range) & $15(0-36)$ \\
back pain & \\
mean preop VAS score (range) & $55(44-80)$ \\
mean postop VAS score (range) & $16(2-38)$ \\
ODI & \\
mean preop score (range) & \\
mean postop score (range) &
\end{tabular}




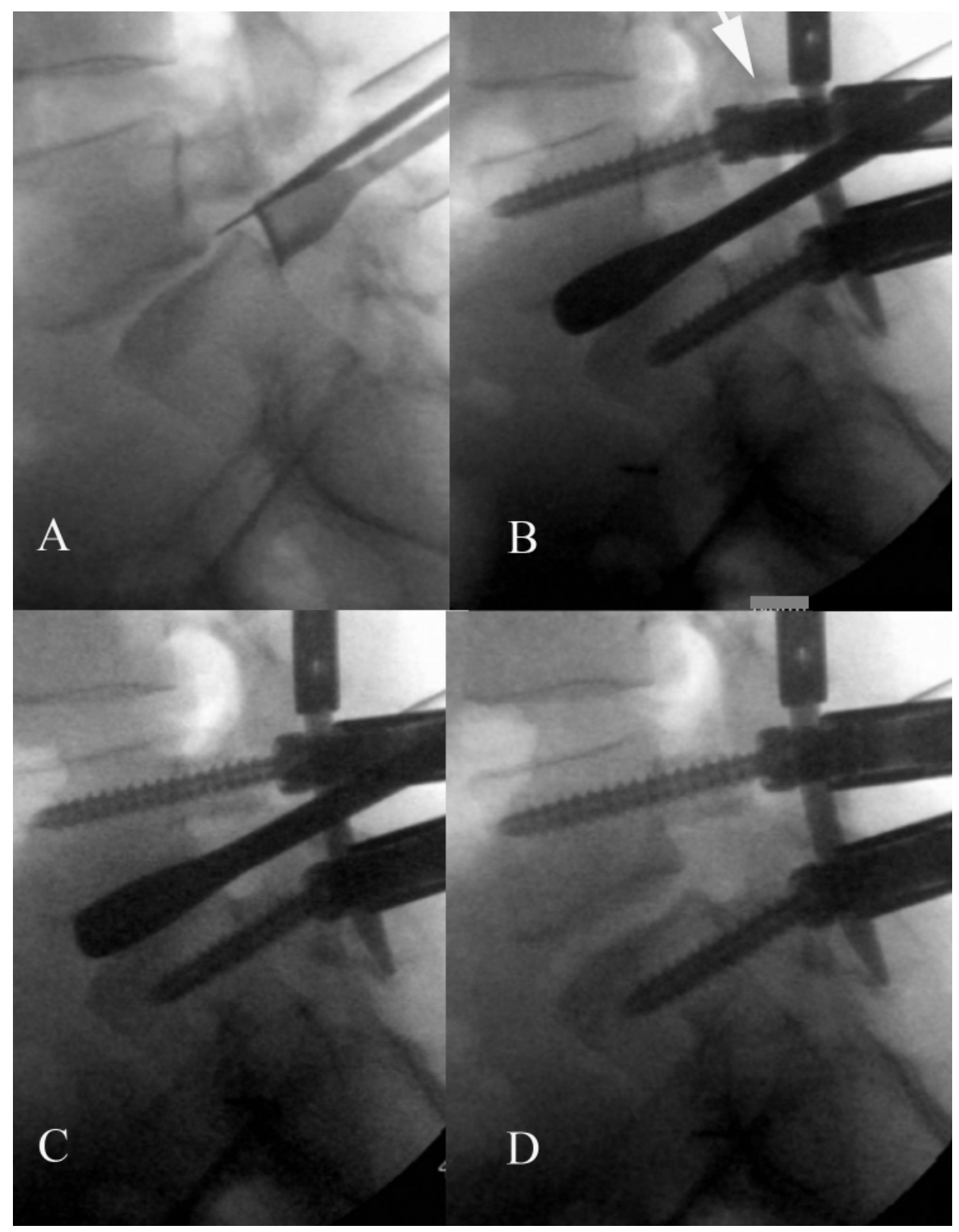

FIG. 6. Lateral fluoroscopic images showing reduction of a spondylolisthesis using the reduction screw extender. Panel B illustrates the initial offset of the rod to the cephalad screw head (arrowhead). Note that the inner sleeve of the caudal screw extender has been adjusted to the non-extended ("reduced") position so that there is no offset of the rod to the caudal screw head. The caudal screw serves as the fixed point during this cantilever-based reduction process. Translation of the cephalad inner sleeve pulls its attached screw head to the rod, reducing the spondylolisthesis (B-D).

improving to 14 and 45.7 at 1-year follow-up. These postoperative outcomes were significantly better than those observed in the laminectomy alone subgroup. In another study, Wu et al. ${ }^{16}$ evaluated outcomes in 82 patients who underwent laminectomy and instrumented posterolateral fusion for degenerative spondylolisthesis and had been subsequently followed up for a minimum of 2 years. The mean preoperative ODI score was 56, decreasing to a mean of 30 at last follow-up. Similarly, in a prospective randomized study comparing posterolateral fusion to conservative therapy for isthmic spondylolisthesis, Möller and Hedlund ${ }^{9}$ found significant improvement in the pain index (average of 2 pain VAS scores) and the Disability Rating Index (DRI). In the 77 cases evaluated, the preoperative pain index and DRI were 63 and 48, respectively, decreasing to 37 and 29 at 2-year follow-up.

In evaluating the outcomes of interbody fusion, Sears ${ }^{15}$ reviewed 34 cases involving patients who underwent open posterior lumbar interbody fusion for the treatment of degenerative spondylolisthesis. Preoperative VAS (0-10 scale), Low Back Outcome scores, and SF-12 scores (physical component summary/mental component summary) were 5.3, 25.1, and 27.9/49.9, respectively, and improved to $2.2,45.2$, and $38.8 / 55.1$ postoperatively with a mean fol- 


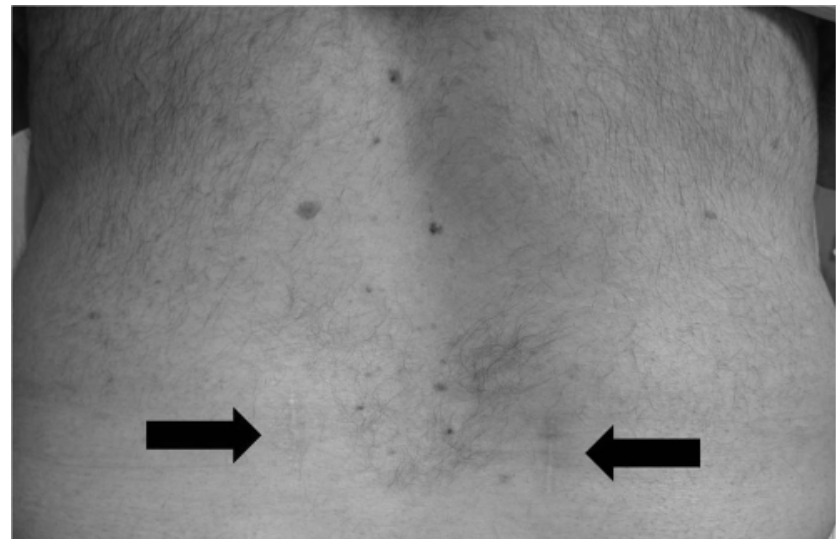

FIG. 7. Photograph showing the skin incisions required for MITLIF (arrows).

low-up of 21.4 months. Lauber et al. ${ }^{7}$ studied 39 cases involving patients treated with open TLIF for spondylolisthesis. Nineteen cases were degenerative, 19 were isthmic, and 1 was dysplastic. In the isthmic/dysplastic subgroup at 2-year follow-up, the mean VAS (0-10 scale) and ODI (0-50 scale) scores were 3.65 and 10.95 , respectively, compared to preoperative scores of 7.15 and 20.05. In the subgroup of patients with degenerative disease, the VAS and ODI scores had also decreased from preoperative values of 8.05 and 27.6, respectively, to postoperative scores of 5.42 and 20.0. Of note, 4-year follow-up scores were reported for 7 patients in the degenerative subgroup; the mean VAS and ODI scores had increased to 6.8 and 21.1, respectively. This increase was attributed to further aging of the spine rather than recurrent symptoms at the previously treated segment.

Although MI-TLIF is a relatively new technical advancement, recent studies suggest that the procedure is as effective as open TLIF in the treatment of degenerative disc disease and/or spondylolisthesis. Schwender et al. ${ }^{14}$ reported a $100 \%$ fusion rate and significantly improved outcomes in 49 patients ( 22 with spondylolisthesis) at 1 year, with the VAS and ODI scores decreasing to 2.2 and 18, respectively, from preoperative values of 7.2 and 46 . In another study, Scheufler et al. ${ }^{13}$ compared 53 patients undergoing MITLIF to a historical cohort of 67 patients who underwent a mini-open TLIF. Thirty-four of the 53 patients were treated for degenerative spondylolisthesis. At 16-month follow-up, intermediate clinical success was noted based on the Roland-Morris low back pain scores and the American Academy of Orthopedic Surgeons lumbar spine questionnaires. The results were similar between the MI-TLIF and mini-open groups, suggesting equivalent outcomes.

In our study, significant improvements in ODI and VAS scores were also noted. The preoperative mean ODI score of 55, signifying moderate disability, decreased to a postoperative mean of 16 , reflecting minimal disability. The leg and back pain VAS scores improved from preoperative means of 65 and 52, respectively, to postoperative means of 8 and 15. With a minimum follow-up of 24 months, symptomatic improvement appears durable and our follow-up period represents one of the longest to date for studies of MI-TLIF. Our results suggest that the clinical outcomes of MI-TLIF compare favorably to outcomes reported for the

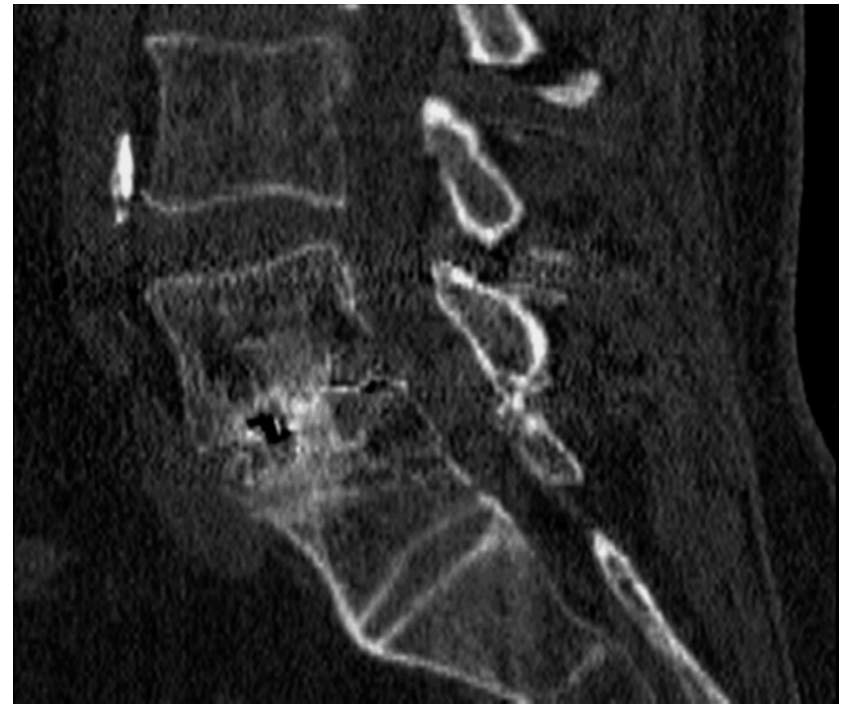

FIG. 8. Sagittal reformatted CT showing interbody fusion.

equivalent open procedures, with the added benefit of minimizing adjacent tissue injury from the minimally invasive approach.

In this paper we have also presented a detailed guideline for performing MI-TLIF and highlighted nuances that we believe will assist surgeons in performing the operation. In all MI-TLIF procedures, determining the ideal entry point and trajectory for optimal placement of the tubular retractor will provide adequate exposure and minimize the need to move the retractor during the operation.

In cases of spondylolisthesis, as is true for open procedures, several methods are employed to achieve reduction during MI-TLIF. Postural reduction occurs with proper patient positioning on the operating table. In cases of degenerative spondylolisthesis, distraction of the disc space (distractive reduction) may significantly decrease the degree of slippage. If significant spondylolisthesis persists after positional and distractive reduction, translational reduction afforded by the reduction screw extenders allows the option of further reduction. While the primary goals of surgery for symptomatic spondylolisthesis remain decompression of the compressed neural elements, restoration of foraminal height, and spinal stabilization, the ability to safely perform spondylolisthesis reduction not only improves sagittal balance but also may potentially improve outcomes.

\section{Conclusion}

In the treatment of symptomatic lumbar spondylolisthesis, MI-TLIF appears to be an effective option, yielding clinical outcomes that are comparable to open surgical procedures. Methods for reduction of spondylolisthesis include positional, distractive, and translational techniques. The availability of reduction screw extenders offers the surgeon an opportunity for percutaneous translational reduction of spondylolisthesis.

\section{Disclosure}

The authors are consultants for Medtronic. The screw 
extenders used in this study were part of an evaluation set of instrumentation provided by Medtronic, but no other support was received for this study.

\section{References}

1. Bednar DA: Surgical management of lumbar degenerative spinal stenosis with spondylolisthesis via posterior reduction with minimal laminectomy. J Spinal Disord Tech 15:105-109, 2002

2. Fairbank JC, Couper J, Davies JB, O'Brien JP: The Oswestry low back pain disability questionnaire. Physiotherapy 66:271-273, 1980

3. Foley KT, Holly LT, Schwender JD: Minimally invasive lumbar fusion. Spine 28 (15 Suppl):S26-S35, 2003

4. Ghogawala Z, Benzel EC, Amin-Hanjani S, Barker FG II, Harrington JF, Magge SN, et al: Prospective outcomes evaluation after decompression with or without instrumented fusion for lumbar stenosis and degenerative Grade I spondylolisthesis. J Neurosurg Spine 1:267-272, 2004

5. Hu S, Bradford DS, Transfeldt EE, Cohen M: Reduction of highgrade spondylolisthesis using Edwards instrumentation. Spine 21:367-371, 1996

6. Isaacs RE, Podichetty VK, Santiago P, Sandhu FA, Spears J, Kelly $\mathrm{K}$, et al: Minimally invasive microendoscopy-assisted transforaminal lumbar interbody fusion with instrumentation. J Neurosurg Spine 3:98-105, 2005

7. Lauber S, Schulte TL, Liljenqvist U, Halm H, Hackenberg L: Clinical and radiologic 2-4-year results of transforaminal lumbar interbody fusion in degenerative and isthmic spondylolisthesis grades 1 and 2. Spine 31:1693-1698, 2006

8. Lowe T, Tahernia AD, O'Brien MF, Smith DA: Unilateral transforaminal posterior lumbar interbody fusion (TLIF): indications, technique, and 2-year results. J Spinal Disord Tech 15:31-38, 2002
9. Möller H, Hedlund R: Surgery versus conservative management in adult isthmic spondylolisthesis - a prospective randomized study: part 1. Spine 25:1711-1715, 2000

10. Mummaneni PV, Rodts GE Jr: The mini-open transforaminal lumbar interbody fusion. Neurosurgery 57 (4 Suppl):256-261, 2005

11. Rengachary SS, Balabhandra R: Reduction of spondylolisthesis. Neurosurg Focus 13(1):E2, 2002

12. Rosenberg W, Mummaneni PV: Transforaminal lumbar interbody fusion: technique, complications, and early results. Neurosurgery 48:569-575, 2001

13. Scheufler KM, Dohmen H, Vougioukas VI: Percutaneous transforaminal lumbar interbody fusion for the treatment of degenerative lumbar instability. Neurosurgery 60 (4 Suppl):203-212, 2007

14. Schwender J, Holly LT, Rouben DP, Foley KT: Minimally invasive transforaminal lumbar interbody fusion (TLIF). J Spinal Disord Tech 18 Suppl:S1-S6, 2005

15. Sears W: Posterior lumbar interbody fusion for degenerative spondylolisthesis: restoration of sagittal balance using insert-and-rotate interbody spacers. Spine J 5:170-179, 2005

16. Wu CH, Kao YH, Yang SC, Fu TS, Lai PL, Chen WJI: Supplementary pedicle screw fixation in spinal fusion for degenerative spondylolisthesis in patients aged 65 and over: outcome after a minimum of 2 years follow-up in 82 patients. Acta Orthop 79:67-73, 2008

Manuscript submitted April 15, 2008.

Accepted May 30, 2008.

Address correspondence to: Paul Park, M.D., Department of Neurosurgery, University of Michigan Health System, 1500 East Medical Center Drive, Room 3552 Taubman Center, Ann Arbor, Michigan 48109-5338. email: ppark@umich.edu. 